\title{
Photodissociation Dynamics of the Thiophenoxy Radical at 248, 193, and $157 \mathrm{~nm}$
}

\author{
Aaron W. Harrison, Jeong Sik Lim, ${ }^{\dagger}$ Mikhail Ryazanov, Gregory Wang, Shumin Gao, ${ }^{\ddagger}$ \\ and Daniel M. Neumark* \\ Department of Chemistry, University of California, Berkeley, California 94720, United States \\ Chemical Sciences Division, Lawrence Berkeley National Laboratory, Berkeley, California 94720, United States
}

Supporting Information

ABSTRACT: The photodissociation dynamics of the thiophenoxy radical $\left(\mathrm{C}_{6} \mathrm{H}_{5} \mathrm{~S}\right)$ have been investigated using fast beam coincidence translational spectroscopy. Thiophenoxy radicals were produced by photodetachment of the thiophenoxide anion followed by photodissociation at $248 \mathrm{~nm}(5.0 \mathrm{eV}), 193 \mathrm{~nm}(6.4 \mathrm{eV})$, and $157 \mathrm{~nm}(7.9 \mathrm{eV})$. Experimental results indicate two major competing dissociation channels leading to $\mathrm{SH}+$ $\mathrm{C}_{6} \mathrm{H}_{4}$ (o-benzyne) and $\mathrm{CS}+\mathrm{C}_{5} \mathrm{H}_{5}$ (cyclopentadienyl) with a minor contribution of $\mathrm{S}+$ $\mathrm{C}_{6} \mathrm{H}_{5}$ (phenyl). Photofragment mass distributions and translational energy distributions were measured at each dissociation wavelength. Transition states and minima for each reaction pathway were calculated using density functional theory to facilitate experimental interpretation. The proposed dissociation mechanism involves internal conversion from the

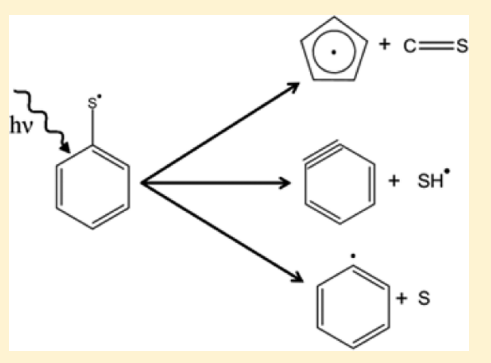
initially prepared electronic excited state to the ground electronic state followed by statistical dissociation. Calculations show that $\mathrm{SH}$ loss involves a single isomerization step followed by simple bond fission. For both SH and S loss, C-S bond cleavage proceeds without an exit barrier. By contrast, the CS loss pathway entails multiple transition states and minima as it undergoes five membered ring formation and presents a small barrier with respect to products. The calculated reaction pathway is consistent with the experimental translational energy distributions in which the CS loss channel has a broader distribution peaking farther away from zero than the corresponding distributions for SH loss.

\section{INTRODUCTION}

Sulfur-containing molecules are a major constituent of both fossil fuels and biomass, and the combustion of these materials results in the emission of many sulfur oxide $\left(\mathrm{SO}_{x}\right)$ pollutants. ${ }^{1}$ Gas phase reactions of sulfur-containing radicals are of particular importance, as these highly reactive species can act as key intermediates in hydrocarbon combustion and have a major influence on the ultimate reaction products. ${ }^{2,3}$ It is thus of considerable interest to characterize the bond dissociation energies of these radicals and to identify the products from their unimolecular decomposition. Molecular beam photodissociation experiments provide a direct probe of the energetics and primary photochemistry of these species, thereby yielding a deeper understanding of their reactivity and their role in complex reaction mechanisms. Herein, we investigate the photodissociation of the thiophenoxy radical $\left(\mathrm{C}_{6} \mathrm{H}_{5} \mathrm{~S}\right)$ using fast beam coincidence translational spectroscopy at 248,193 , and $157 \mathrm{~nm}$.

This work is motivated in part by recent photodissociation experiments on the aromatic sulfur-containing compounds thiophenol $\left(\mathrm{C}_{6} \mathrm{H}_{5} \mathrm{SH}\right)$ and thioanisole $\left(\mathrm{C}_{6} \mathrm{H}_{5} \mathrm{SCH}_{3}\right)$. These species are important model systems for gaining a general understanding of the role played by conical intersections in dictating the photodissociation dynamics of aromatic molecules. Translational energy distributions for the $\mathrm{H}$ atom and $\mathrm{CH}_{3}$ loss channels were determined using velocity map ion imaging $^{4-7}$ and $\mathrm{H}$-atom Rydberg tagging. ${ }^{8-10}$ From these distributions, one obtains the branching ratio between the ground state and first excited state of the thiophenoxy photofragment, thereby probing the nonadiabatic couplings among the parent electronic states as dissociation proceeds through various conical intersections.

While the kinetics and reaction mechanisms involved in the production of thiophenoxy have been examined in some detail in the gas phase ${ }^{4-10}$ and in solution, ${ }^{11-16}$ its photochemistry is less well-characterized. Experiments in solution ${ }^{13,17}$ and gas phase $^{18,19}$ have attributed two major absorption bands near 2.48 and $4.13 \mathrm{eV}$ to thiophenoxy with tentative assignments of the transitions involved. Further study of thiophenoxy using laser-induced fluorescence following the photolysis of thiophenol and thioanisole found the origin of the first bright state to be $2.397 \mathrm{eV}^{20}$ this band was assigned to the $\tilde{B}^{2} \mathrm{~A}_{2}-X^{2} \mathrm{~B}_{1}$ transition. Recently, slow electron velocity-map imaging of the thiophenoxide anion has provided accurate values of both the adiabatic electron affinity $(2.3542 \mathrm{eV})$ and the energy gap $(0.3719 \mathrm{eV})$ between the $\tilde{\mathrm{A}}$ and $\tilde{\mathrm{X}}$ states of thiophenoxy. ${ }^{21}$

It is of interest to compare the thiophenoxy radical to the more thoroughly studied phenoxy radical $\mathrm{C}_{6} \mathrm{H}_{5} \mathrm{O}$. The thermal

Special Issue: Curt Wittig Festschrift

Received: April 1, 2013

Revised: July 1, 2013

Published: July 3, 2013 
decomposition of phenoxy has been the subject of numerous experimental and theoretical studies owing to its central importance in the combustion of aromatic compounds. ${ }^{22-26}$ The only observed fragmentation pathway of phenoxy involves rearrangement of the ring system to form cyclopentadienyl radical $\left(\mathrm{C}_{5} \mathrm{H}_{5}\right)$ and carbon monoxide $(\mathrm{CO})$; it was determined that the mechanism for this reaction proceeds by initial formation of a bicyclic intermediate followed by the elimination of carbon monoxide from the remaining cyclopentadienyl ring. ${ }^{23,24}$ While this pathway presents a barrier to the formation of products, the thermodynamic stability of these products leads to a small $\Delta H^{\circ}(\sim 1 \mathrm{eV})$ for the overall reaction. Other possible dissociation channels such as hydroxyl radical and ortho-benzyne formation or oxygen loss to form phenyl radical and $\mathrm{O}$ atom are substantially higher in energy $(\sim 5 \mathrm{eV})$ and do not compete with CO loss as a thermal decomposition channel.

The corresponding dissociation channels of thiophenoxy ${ }^{27,28}$ are much closer in energy:

$$
\begin{array}{clrl}
\mathrm{C}_{6} \mathrm{H}_{5} \mathrm{~S} & \longrightarrow \mathrm{C}_{5} \mathrm{H}_{5}(\text { cyclopentadienyl })+\mathrm{CS} & D_{\mathrm{o}}=3.21 \pm 0.15 \mathrm{eV} \\
\longrightarrow \mathrm{C}_{6} \mathrm{H}_{4}(o-\text { benzyne })+\mathrm{SH} & D_{\mathrm{o}}=3.60 \pm 0.10 \mathrm{eV} \\
\longrightarrow \mathrm{C}_{6} \mathrm{H}_{5}+\mathrm{S} & D_{\mathrm{o}}=3.90 \pm 0.10 \mathrm{eV}
\end{array}
$$

The enthalpy of formation of the cyclopentadienyl radical is still a topic of some controversy with a range of values from 2.5 to $2.8 \mathrm{eV}$ reported in the last 30 years. More recent studies have favored the higher end of this range, and so to estimate the dissociation energy for eq 1 , we used a value of $2.75 \mathrm{eV}^{29}$

Herein, we report results on the photodissociation of the thiophenoxy radical at 248,193 , and $157 \mathrm{~nm}$ prepared by nearthreshold photodetachment of the corresponding anion, thiophenoxide. We obtain translational energy and photofragment angular distributions, $P\left(E_{\mathrm{T}}, \theta\right)$, as well as branching ratios for the competing dissociation channels. These data indicate a statistical dissociation mechanism where absorption to an electronically excited state is followed by internal conversion to the ground state where dissociation occurs. In addition, we employ density functional theory (DFT) calculations and Rice-Ramsperger-Kassel-Marcus (RRKM) modeling to support our experimental findings.

\section{EXPERIMENTAL SECTION}

The fast beam coincidence translational spectrometer used in this study has been described in detail elsewhere, ${ }^{30,31}$ but a new detection system has been recently added and is discussed below. In this experiment, a fast beam of mass-selected thiophenoxide anions $\left(\mathrm{C}_{6} \mathrm{H}_{5} \mathrm{~S}^{-}\right)$is generated and photodetached to make thiophenoxy radicals. The radicals are dissociated by a second laser, and the neutral fragments are detected in coincidence:

$$
\mathrm{C}_{6} \mathrm{H}_{5} \mathrm{~S}^{-} \stackrel{\mathrm{h} v_{1}}{\longrightarrow} \mathrm{C}_{6} \mathrm{H}_{5} \mathrm{~S} \stackrel{\mathrm{h} v_{2}}{\rightarrow} \text { fragments }
$$

Here, an $\sim 0.1 \%$ thiophenol/He mixture at 50 psi is expanded into the vacuum chamber through a pulsed molecular beam valve equipped with a DC discharge source to produce thiophenoxide anions. Resulting anions are then accelerated to a beam energy of $5 \mathrm{keV}$ and mass-selected using a Bakkertype mass spectrometer ${ }^{32,33}$ that imparts negligible kinetic energy spread to the ion beam. Mass-selected thiophenoxide anions are then photodetached at $518 \mathrm{~nm}(2.394 \mathrm{eV})$ with an excimer-pumped dye laser (Lambda-Physik LPX-200 and Lambda-Physik FL 3002). This detachment energy is slightly above the electron affinity for thiophenoxy, $2.3542 \mathrm{eV}^{21}$ so as to minimize the production of vibrationally excited radicals. Those ions that remain are deflected from the beam, leaving behind a fast beam of neutral thiophenoxy radicals. This neutral packet is intersected by a UV pulse from a second excimer laser (GAM EX-50F) at $248 \mathrm{~nm}(5.0 \mathrm{eV}), 193 \mathrm{~nm}(6.4 \mathrm{eV})$, or 157 $\mathrm{nm}(7.9 \mathrm{eV})$. Photofragments from dissociated radicals recoil off the beam axis and strike a time- and position-sensitive (TPS) detector located $2 \mathrm{~m}$ downstream from the laser interaction region, while undissociated radicals are blocked by a $5 \mathrm{~mm}$ radius beam block in front of the detector.

We perform coincidence measurements in which the positions on the detector and differences of arrival times are determined for both photofragments from each dissociation event, thereby yielding the translational energy release, scattering angle, and photofragment mass ratio for each event. $^{34,35}$ From these, we can construct the translational energy and photofragment angular distribution, $P\left(E_{\mathrm{T}}, \theta\right)$, given by the following uncoupled relation

$$
P\left(E_{\mathrm{T}}, \theta\right)=P\left(E_{\mathrm{T}}\right) \cdot\left[1+\beta\left(E_{\mathrm{T}}\right) P_{2}(\cos \theta)\right]
$$

where $\beta$ is the energy-dependent anisotropy parameter and $P_{2}$ is the second order Legendre polynomial. For this experiment, $\theta$ is defined as the angle of the dissociation recoil axis with respect to the laser propagation direction as the unpolarized output from our GAM excimer laser was used for photodissociation. In this case, $\beta$ takes on the values between -1 and $1 / 2$ for parallel and perpendicular transitions, respectively. ${ }^{36}$

We have recently upgraded our TPS detection scheme to a Roentdek Hex80 delay line anode detector. ${ }^{37}$ Our previous TPS detector involved a microchannel plate (MCP) assembly with a phosphor screen and a charge-coupled device (CCD) camera for accurate position measurement and $4 \times 4$ multianode photomultiplier tube (PMT) array for accurate timing and rough position information. ${ }^{31}$ The delay-line anode has the advantage of much simpler data acquisition and readout while requiring fewer detection components and less alignment for operation. The previous setup also had some limitations on timing recognition of neighboring particle hits that were encoded by the same PMT anode. While uncommon with traditional 2-body dissociation, dissociation into three (or more) fragments ${ }^{38,39}$ can result in two fragments read by the same PMT anode, resulting in a discarded data point. In contrast, the delay line anode allows more efficient collection of events with small interfragment separation.

The Roentdek hexanode and the principles underlying delay line detection have been described in detail elsewhere. ${ }^{37,40,41}$ The detector comprises a Z-stack of three $75 \mathrm{~mm}$ diameter MCPs with a 40:1 aspect ratio mounted to a delay line anode consisting of three layers of delay lines. When an incident particle strikes the front MCP, an electron cascade is produced, and the resulting charge cloud that emerges from the MCP stack is collected by the three-layered anode. Each layer comprises one delay line along which the end-to-end signal transit time is constant $\left(T_{\text {sum }} \sim 100 \mathrm{~ns}\right)$. Measuring the relative delay between the arrival times $t_{1}$ and $t_{2}$ of the counterpropagating signal pulse at each end of the wire yields one dimension of the particle position, e.g., $x \propto t_{1}-t_{2}$. From any two of the layers, the two-dimensional (2D) position of the article is determined. The third layer offers redundant position information, minimizing the "dead zone" of the detector and allowing for reconstruction of data points when signal is lost or overlapped at the end of the delay line. The corresponding particle arrival times are obtained directly from the MCP stack. 

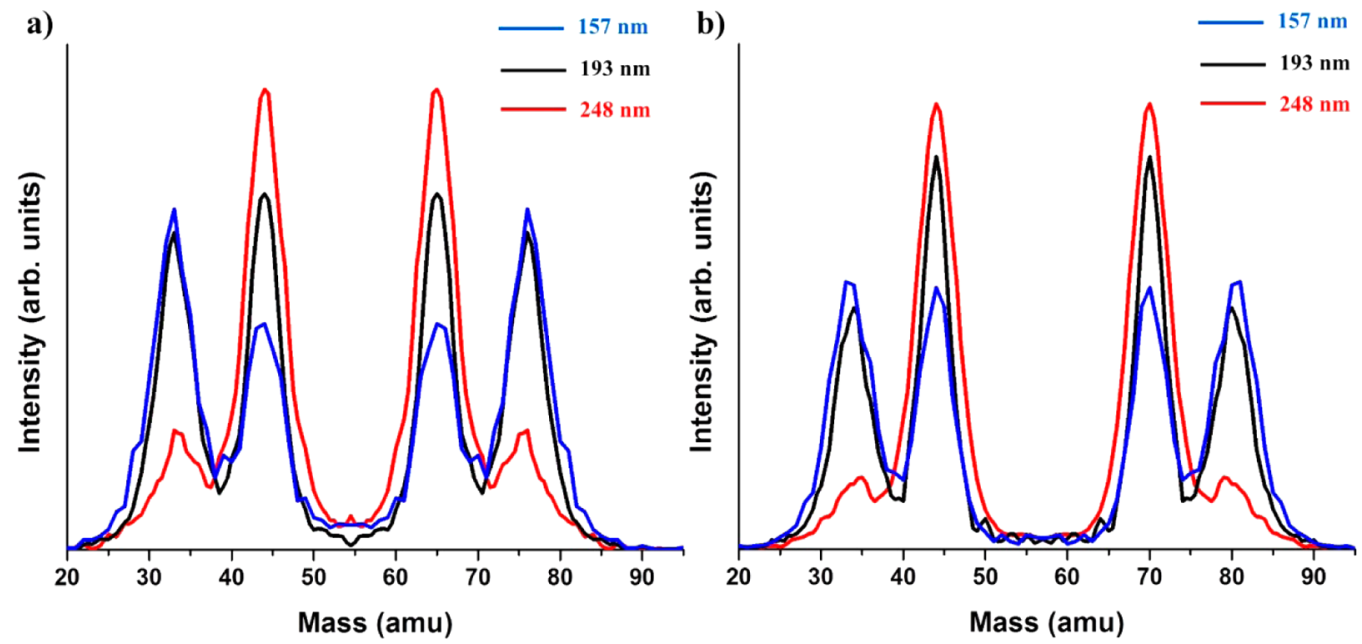

Figure 1. Raw photofragment mass spectra of (a) $\mathrm{C}_{6} \mathrm{H}_{5} \mathrm{~S}$ and (b) $\mathrm{C}_{6} \mathrm{D}_{5} \mathrm{~S}$ at $248 \mathrm{~nm}$ (red), $193 \mathrm{~nm}$ (black), and $157 \mathrm{~nm}$ (blue).

MCP and delay line signals are sent to a fast amplifier (FAMP, Roentdek) and consecutively transformed to a standard NIM pulse using a constant fraction discriminator (CFD8, Roentdek). Finally, these NIM pulses are fed into a time-todigital convertor (TDC8, Roentdek) to be written into onboard memory. The time and position resolution are estimated to be $100 \mathrm{ps}$ and $100 \mu \mathrm{m}$, respectively. Based on calibration experiments using the predissociation from the $B$ state of $\mathrm{O}_{2}{ }^{42}$ the kinetic energy resolution $(\Delta E / E)$ is measured to be $\sim 0.8 \%$. Further information about the detector resolution is provided in the Supporting Information (SI-1).

In our experimental setup, the presence of the beam block and the finite radius of the detector lead to lower detection efficiency and larger error in the measured intensity for events with very low and high translational energies, respectively; this effect depends on the photofragment scattering angle as well. To account for these effects, the raw translational energy distributions are adjusted with a detector acceptance function (DAF). ${ }^{35}$ The $P\left(E_{\mathrm{T}}\right)$ distributions and branching ratios presented here have all been corrected using the DAF.

\section{RESULTS AND ANALYSIS}

A. Photofragment Mass and Translational Energy Distributions. Using conservation of momentum, the product mass ratio is determined from the measured distance of the two fragments from the dissociation center at the detector. This leads to a fragment mass resolution that is mainly dependent on the diameter of the parent beam at the detector. The radical beam is on the order of $1 \mathrm{~mm}$ at full width half-maximum (fwhm) yielding a fragment mass resolution $(m / \Delta m)$ of $\sim 10 .^{43}$

Due to the relatively low mass resolution on the photofragments, we examined the photodissociation of both $\mathrm{C}_{6} \mathrm{H}_{5} \mathrm{~S}$ and $\mathrm{C}_{6} \mathrm{D}_{5} \mathrm{~S}$ to better differentiate between $\mathrm{S}$ and $\mathrm{SH}$ loss. Figure 1 shows product mass distributions at $248 \mathrm{~nm}(5.0 \mathrm{eV}), 193 \mathrm{~nm}$ $(6.4 \mathrm{eV})$, and $157 \mathrm{~nm}(7.9 \mathrm{eV})$ for both the undeuterated and deuterated species with Table 1 listing the corresponding contributions for each mass channel and dissociation wavelength. For the undeuterated species, these distributions have peak masses near $44+65$ (mass channel B) corresponding to CS loss (eq 1) and $33+76$ (mass channel A). Mass channel A is closer in mass to the $\mathrm{SH}$ loss channel (eq 2) but could also be due to $\mathrm{S}$ loss (eq 3 ) or some mixture thereof. If $\mathrm{SH}$ loss is the major dissociation channel, the peak for the lightest photofrag-
Table 1. Relative Contributions of Mass Channels A and B for $\mathrm{C}_{6} \mathrm{H}_{5} \mathrm{~S}$ and $\mathrm{C}_{6} \mathrm{D}_{5} \mathrm{~S}$ at Each Dissociation Wavelength ${ }^{a}$

$\begin{array}{ccccc}\text { wavelength } & \begin{array}{c}\mathrm{C}_{6} \mathrm{H}_{5} \mathrm{~S} \\ \text { mass } \\ \text { channel A }\end{array} & \begin{array}{c}\mathrm{C}_{6} \mathrm{H}_{5} \mathrm{~S} \\ \text { mass } \\ \text { channel B }\end{array} & \begin{array}{c}\mathrm{C}_{6} \mathrm{D}_{5} \mathrm{~S} \\ \text { mass } \\ \text { channel A }\end{array} & \begin{array}{c}\mathrm{C}_{6} \mathrm{D}_{5} \mathrm{~S} \\ \text { mass } \\ \text { channel B }\end{array} \\ 248 \mathrm{~nm} & 23 \% & 77 \% & 18 \% & 82 \% \\ 193 \mathrm{~nm} & 48 \% & 52 \% & 42 \% & 58 \% \\ 157 \mathrm{~nm} & 59 \% & 41 \% & 53 \% & 47 \%\end{array}$

${ }^{a}$ Error in these values is estimated to be $\pm 1 \%$.

ment in the mass distribution of the deuterated species should shift to slightly higher mass. However for $S$ loss, this peak's position should be relatively unchanged. In Figure 2, the mass distributions for the lighter photofragment of mass channels $\mathrm{A}$ and $B$ are overlaid at each wavelength for $\mathrm{C}_{6} \mathrm{H}_{5} \mathrm{~S}$ and $\mathrm{C}_{6} \mathrm{D}_{5} \mathrm{~S}$. We observe a small shift to higher mass for mass channel $\mathrm{A}$, while for mass channel $\mathrm{B}$ the peak position is unchanged. This observation is consistent with $\mathrm{SH} / \mathrm{SD}$ and $\mathrm{CS}$ formation. Furthermore, there is also a small isotope effect where deuteration leads to less population of mass channel A by comparison to the undeuterated species.

To gain more insight into the relative contributions of $\mathrm{SH}$ and $\mathrm{S}$ loss to mass channel $\mathrm{A}$, the mass distributions at each wavelength were simulated using the experimental conditions and translational energy distributions (discussed below) with varying contributions of the possible product masses. At 248 and $193 \mathrm{~nm}$, the best fit is obtained using mostly SH/SD, but the intensity of mass channel A is very low at $248 \mathrm{~nm}$ leading to more error in the simulation. At $157 \mathrm{~nm}$, better fits are obtained when using approximately $2.5: 1$ of $\mathrm{SH}$ to $\mathrm{S}$. A sample simulation is included in the Supporting Information (SI-2) with estimations of the branching ratios obtained from this method. In addition, kinetics calculations discussed in Section 3C further indicate that SH loss dominates over S loss at 248 and $193 \mathrm{~nm}$ and that $\mathrm{S}$ loss becomes more competitive at 157 $\mathrm{nm}$.

It is clear that for both $\mathrm{C}_{6} \mathrm{H}_{5} \mathrm{~S}$ and $\mathrm{C}_{6} \mathrm{D}_{5} \mathrm{~S}$, CS loss (mass channel B) dominates at $248 \mathrm{~nm}$, but $\mathrm{SH} / \mathrm{SD}$ loss (mass channel A) is much more prominent at $193 \mathrm{~nm}$. At $157 \mathrm{~nm}$, the combination of $\mathrm{SH}$ and $\mathrm{S}$ loss in mass channel $\mathrm{A}$ has more population than the CS loss channel. On the basis of the relative contributions of the two mass channels listed in Table 1 , we find the branching ratio of mass channels $A: B$ to be 

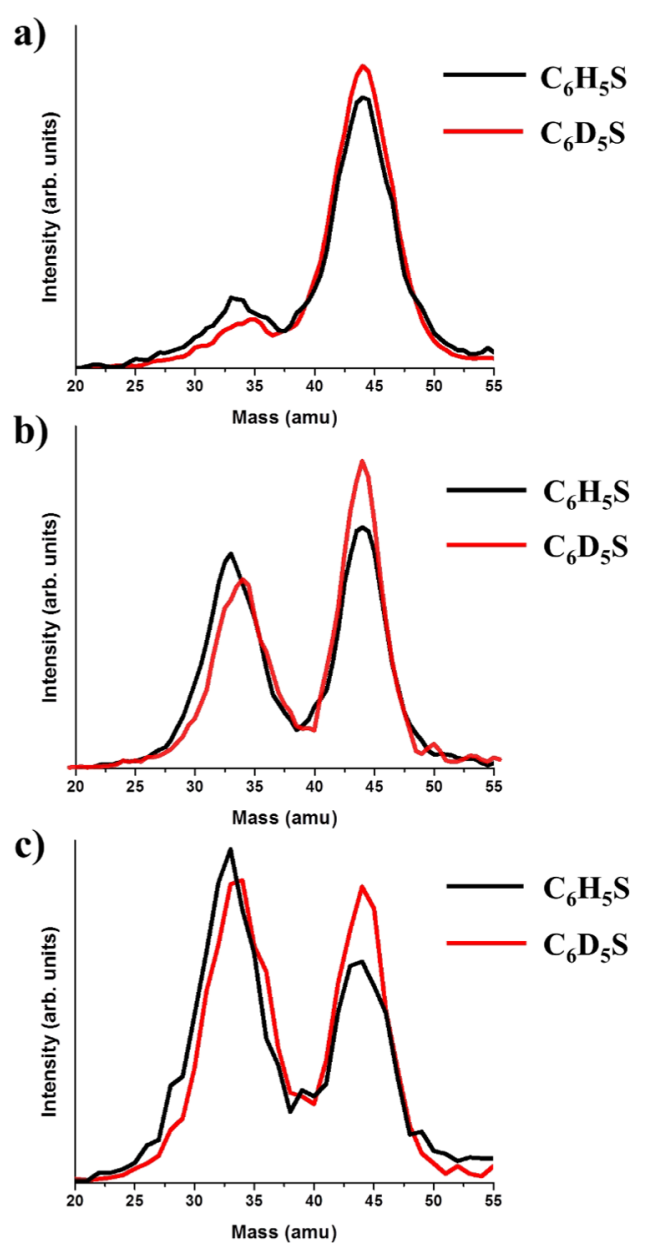

Figure 2. Overlays of the mass spectra for the light photofragments of $\mathrm{C}_{6} \mathrm{H}_{5} \mathrm{~S}$ (black) and $\mathrm{C}_{6} \mathrm{D}_{5} \mathrm{~S}$ (red) at (a) $248 \mathrm{~nm}$, (b) $193 \mathrm{~nm}$, and (c) $157 \mathrm{~nm}$.

0.30:1, 0.92:1, and 1.44:1 for dissociation at 248,193, and 157 nm, respectively. For $\mathrm{C}_{6} \mathrm{D}_{5} \mathrm{~S}$, the branching ratios are slightly smaller at every photon energy with values of $0.22: 1,0.72: 1$, and 1.13:1.

Figure 3 shows the corresponding translational energy distributions for each fragment mass channel and dissociation wavelength. From energy conservation, the translational energy release of the photofragments, $E_{\mathrm{T}}$, is given by the following relationship:

$$
E_{\mathrm{T}}=h v-E_{\mathrm{int}}-D_{\mathrm{o}}
$$

where $h v$ is the photon energy, $E_{\text {int }}$ is the internal energy of fragments, and $D_{\mathrm{o}}$ is the bond dissociation energy. Equation 6 assumes that the precursor radicals have no internal energy, a reasonable assumption since the anions are photodetached just above threshold (Section 2).

Because $\mathrm{SH}$ and $\mathrm{S}$ loss are unresolved in the mass distributions, we cannot make distinct translational energy distributions for the two product channels. As such, mass channel A is treated as $\mathrm{SH}$ loss for the following analysis, but there may be also a small contribution from $S$ loss especially at $157 \mathrm{~nm}$. Also, the translational energy distributions for these dissociation channels are expected to be similar because both channels lack an exit barrier (Section 3B) and have nearly equal available energy.

At $248 \mathrm{~nm}$, the maximum kinetic energy available to the fragments is 1.4 and $1.8 \mathrm{eV}$ for the $\mathrm{SH}$ loss and CS loss channels, respectively. The translational energy distribution for the CS loss channel peaks at $0.22 \mathrm{eV}$ and extends out to approximately $1 \mathrm{eV}$. For $\mathrm{SH}$ loss, the $P\left(E_{\mathrm{T}}\right)$ distribution peaks closer to zero $(0.10 \mathrm{eV})$ and is sharper with virtually all of its intensity below $0.5 \mathrm{eV}$. Similarly, at 193 and $157 \mathrm{~nm}$, the translational energy distribution for the CS loss channel is broader than that of the SH loss channel, but at the higher photon energies, the distributions are broader and extend out to higher translational energy than the corresponding distributions at $248 \mathrm{~nm}$. The maximum available energy $\left(E_{\mathrm{T}}^{\mathrm{Max}}\right)$, peak translational energy $\left(E_{\mathrm{T}}^{\text {Peak }}\right)$, average translational energy release $\left\langle E_{\mathrm{T}}\right\rangle$, and the corresponding fractional average energy release $\left\langle f_{\mathrm{T}}\right\rangle$ for each channel and dissociation energy are listed in Table 2. The translational energy distributions for deuterated thiophenoxy have not been included, as they are very similar to the undeuterated species. Finally, all angular distributions were found to be isotropic, yielding $\beta\left(E_{\mathrm{T}}\right)$ values approximately equal to zero within the experimental uncertainties.

B. Electronic Structure Calculations. To gain further insight into the dissociation dynamics of thiophenoxy, a)

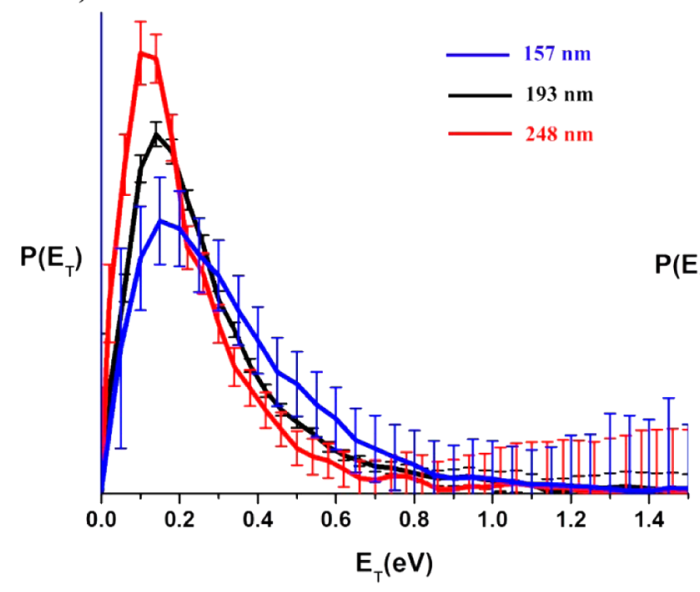

b)

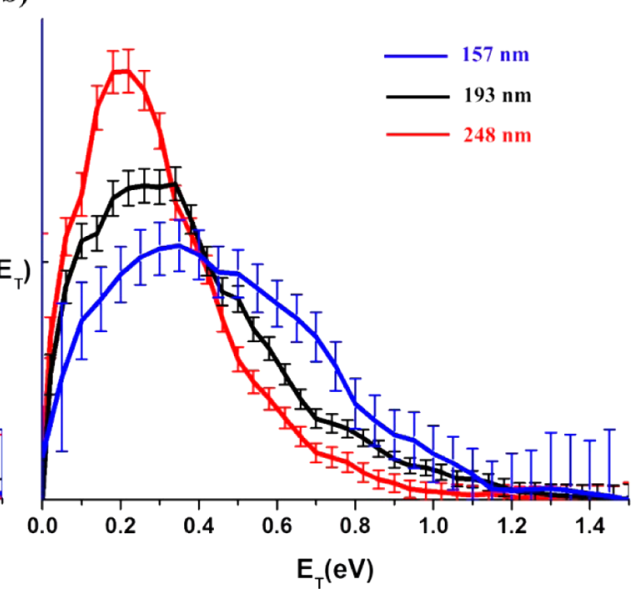

Figure 3. Translational energy distributions (normalized) of (a) $\mathrm{SH}+\mathrm{C}_{6} \mathrm{H}_{4}$ and (b) $\mathrm{CS}+\mathrm{C}_{5} \mathrm{H}_{5}$ dissociation channels at 248 (red), 193 (black), and $157 \mathrm{~nm}$ (blue). 
Table 2. Maximum Available, Peak Translational, and Average Translational Energy Release with the Corresponding Fractional Average Energy Release for Both Product Channels and Dissociation Energies of $5.0 \mathrm{eV}$ (248 $\mathrm{nm}), 6.4 \mathrm{eV}(193 \mathrm{~nm})$, and $7.9 \mathrm{eV}(157 \mathrm{~nm})^{a}$

\begin{tabular}{cccccc} 
& photon energy & $E_{\mathrm{T}}^{\mathrm{Max}}$ & $E_{\mathrm{T}}^{\text {Peak }}$ & $\left\langle E_{\mathrm{T}}\right\rangle$ & $\left\langle f_{\mathrm{T}}\right\rangle$ \\
\hline \multirow{3}{*}{ SH loss } & 5.0 & 1.4 & 0.10 & 0.18 & 0.11 \\
& 6.4 & 2.8 & 0.14 & 0.25 & 0.09 \\
& 7.9 & 4.3 & 0.15 & 0.35 & 0.08 \\
& 5.0 & 1.8 & 0.22 & 0.32 & 0.18 \\
& 6.4 & 3.2 & 0.30 & 0.41 & 0.13 \\
& 7.9 & 4.7 & 0.35 & 0.47 & 0.10
\end{tabular}

${ }^{a}$ All energies are in $\mathrm{eV}$.

electronic structure calculations of the unimolecular reaction intermediates and products involved in each reaction pathway were performed. Previous theoretical studies on the thermal decomposition mechanism of the phenoxy radical indicated the importance of high level electron correlation methods in reliably predicting the energetics of the isomeric intermediates along the thermal decomposition pathway. ${ }^{23,24}$ However, the relative energies of the transition states and intermediates calculated using DFT with the B3LYP functional gave reasonable agreement with higher level methods $(\operatorname{RCCSD}(\mathrm{T})$ and CASPT2) for phenoxy decomposition ${ }^{24}$ and also performed reasonably well in a theoretical study of the ground and first excited state of thiophenoxy. ${ }^{44}$

For this study, all equilibrium structures and transition states were optimized with DFT-B3LYP using the Dunning-type ccpVDZ basis set augmented with diffuse functions in Gaussian 09 . $^{45}$ Minima and transition states were confirmed by frequency analysis and by following the intrinsic reaction coordinate (IRC) with DFT. These calculations were followed by single point energy calculations on the optimized structures using restricted open-shell MP2 (ROMP2) with the aug-cc-pVTZ basis set. Single point calculations at the ROMP2/aug-cc-pVTZ level theory with the DFT geometries were found to order the asymptotic dissociation energies better than DFT, so we chose this level of theory to calculate relative energies of the minima and transition states for each reaction pathway. Figure 4 shows the lowest energy reaction pathways and calculated energetics, including harmonic zero-point energies for both channels.

As is the case for CO loss from phenoxy, ${ }^{23,24}$ there are two possible routes for CS loss. The more energetically facile mechanism proceeds via the bicyclic isomerization pathway (shown in Figure 4) where the $\beta$ carbons approach to form a new bond. The optimized geometries with $\mathrm{C}-\mathrm{C}$ and $\mathrm{C}-\mathrm{S}$ bond lengths $(\AA)$ along with the point group symmetries for the transition states and minimum energy structures for this reaction pathway are presented in Figure 5. In Figures 4 and 5, the primary nuclear motion corresponding to the imaginary frequency is represented by the dotted bond. The higher energy pathway (not shown) occurs by direct ring-opening through homolytic cleavage of the $\mathrm{C}-\mathrm{C}$ bond of $\mathrm{C} 1$ with one of the $\beta$ carbons. Our calculations indicate the initial transition state for the ring-opening pathway to be approximately $1 \mathrm{eV}$ higher in energy than for the bicyclic pathway. Experimental kinetics data and RRKM calculations confirmed the lower energy pathway to be the preferred mechanism for phenoxy, ${ }^{23-25}$ so this is the mechanism we focus on here.

The first step of the CS loss mechanism involves a pericyclic reaction where 1 passes through the first transition state, TS1, calculated to be $2.65 \mathrm{eV}$ above the ground state of thiophenoxy, and then isomerizes to the bicyclic intermediate $2(2.43 \mathrm{eV})$. This is followed by bond extension between $\mathrm{C} 1$ and one of the $\beta$ carbons of 2 to form TS2 $(2.64 \mathrm{eV})$ as the $\mathrm{C} 1-\mathrm{C} 2-\mathrm{C} 6$ ring system begins to open. Continuing along this path results in bond cleavage, leaving $\mathrm{C} 1$ bound to only one of the original $\beta$ carbons to form $3(2.32 \mathrm{eV})$. Finally, the highest energy barrier occurs at TS3 $(3.58 \mathrm{eV})$ as the CS moiety departs from the five-membered ring to yield $\mathrm{CS}+\mathrm{C}_{5} \mathrm{H}_{5}$. The dissociation energy for CS loss was calculated to be $3.37 \mathrm{eV}$, in comparison to the experimental value of $3.2 \mathrm{eV}^{27-29}$

For SH loss, a hydrogen atom from one of the $\beta$ carbons of $\mathbf{1}$ approaches sulfur to form TS4 $(2.40 \mathrm{eV})$ then completes isomerization to the thiol radical intermediate $4(1.44 \mathrm{eV})$. From 4, C-S bond cleavage proceeds without a barrier to produce the $\mathrm{SH}$ radical and ortho-benzyne at a calculated dissociation energy of $3.74 \mathrm{eV}$, slightly larger than the experimental value of $3.6 \mathrm{eV} .^{27}$ The optimized structures for this pathway are also shown in Figure 5. Finally, $\mathrm{S}$ loss proceeds through a pathway with no exit barrier to $\mathrm{C}_{6} \mathrm{H}_{5}+\mathrm{S}$. The

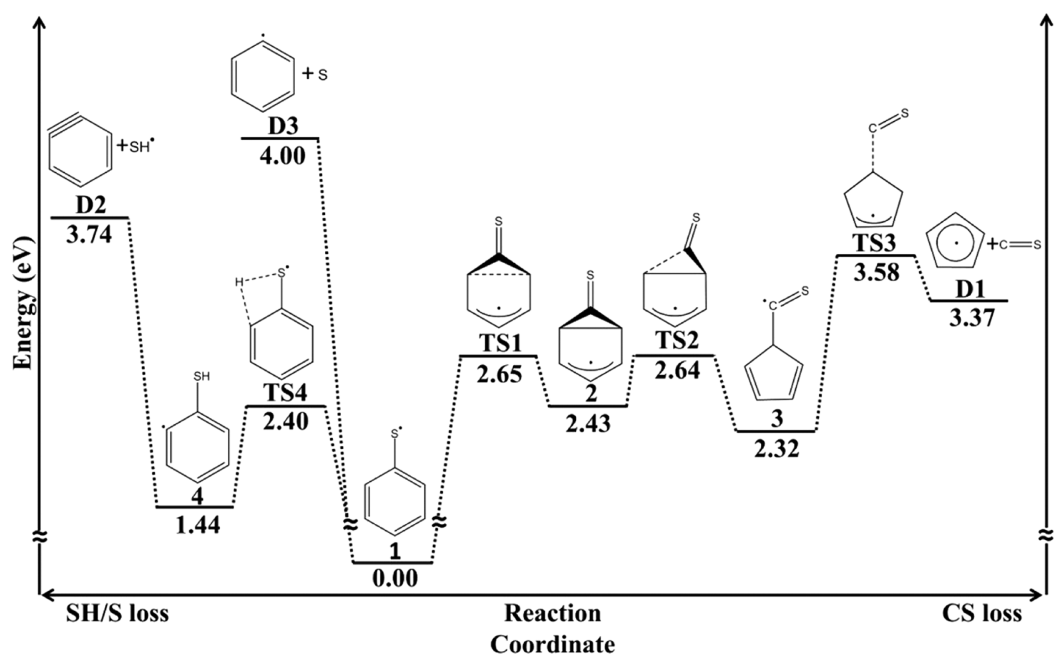

Figure 4. Schematic of the potential energy surfaces for $\mathrm{C}_{6} \mathrm{H}_{5} \mathrm{~S}$ dissociation channels. Energies $(\mathrm{eV})$ are relative to thiophenoxy ground state and calculated at ROMP2/aug-cc-pVDZ + ZPE (B3LYP/aug-cc-pVDZ) level of theory. 

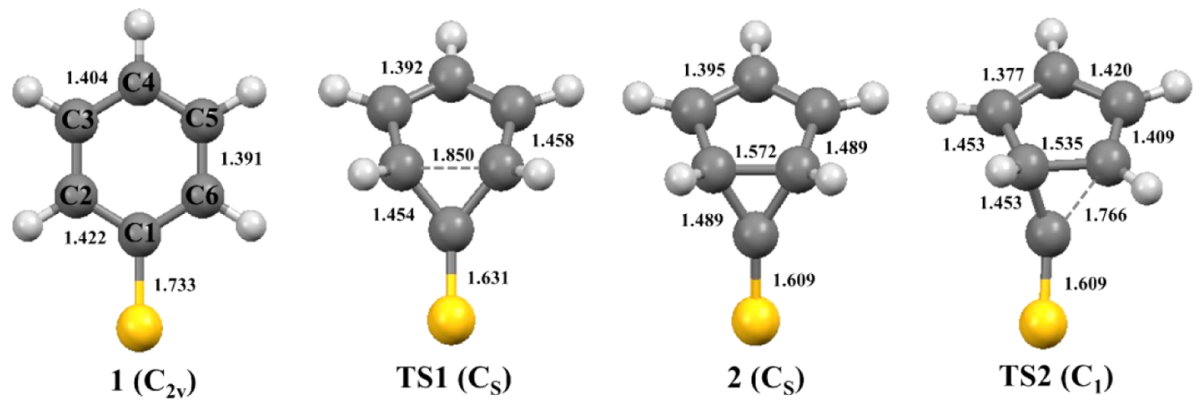

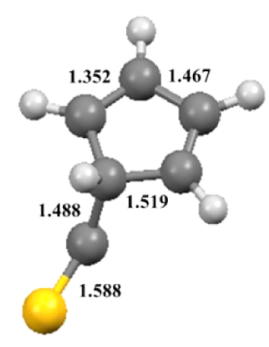

$3\left(\mathrm{C}_{\mathrm{S}}\right)$

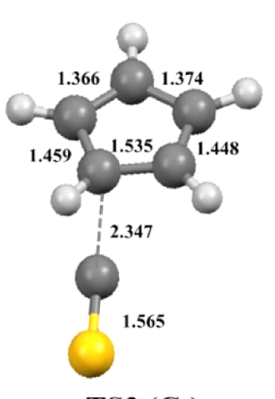

$\operatorname{TS} 3\left(C_{1}\right)$

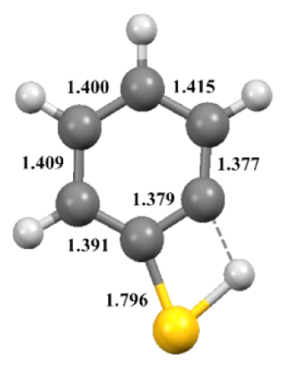

TS4 $\left(\mathrm{C}_{1}\right)$

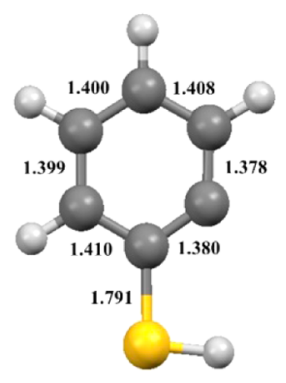

$4\left(C_{1}\right)$

Figure 5. Optimized geometries for the minima and transition states for the CS and SH loss channels (see Figure 4 for labeling). For all structures, $\mathrm{C}-\mathrm{C}$ and $\mathrm{C}-\mathrm{S}$ bond lengths $(\AA)$ and point group symmetries are shown. Dotted bond for transition states indicates the primary nuclear motion corresponding to the imaginary frequency.

dissociation energy was determined to be $4.00 \mathrm{eV}$ in good agreement with the experimental value of $3.9 \mathrm{eV}^{27}$

C. RRKM Calculations. RRKM theory ${ }^{46}$ was used to model the relative kinetics for the competing dissociation channels. The RRKM rate constant, $k(E)$, is given by

$$
k(E)=\frac{W^{\dagger}\left(E-E_{\mathrm{o}}\right)}{h \rho(E)}
$$

Here $W^{\dagger}$ is the sum of states at the critical configuration while $E_{\mathrm{o}}$ represents the relative energy of this configuration with respect to the reactant whose density of states is $\rho(E)$. The sums and densities of states were evaluated using the BeyerSwinehart algorithm ${ }^{47}$ for calculation of the rate constants. All modes other than the reaction coordinate for both channels were treated as harmonic vibrations whose frequencies were taken from the electronic structure calculations.

To find the overall unimolecular rate constant for CS loss, we treated the following reaction mechanism

$$
\mathbf{1} \underset{k_{-1}}{\stackrel{k_{1}}{\rightleftharpoons}} \mathbf{2} \underset{k_{-2}}{\stackrel{k_{2}}{\rightleftharpoons}} \mathbf{3} \stackrel{k_{3}}{\rightarrow} \mathrm{D} 1
$$

by applying a steady-state approximation to intermediates 2 and 3 (Figure 3). This analysis results in the following expression for the rate constant for CS loss

$$
k_{\mathrm{CS} \text { loss }}=\frac{k_{1} k_{2} k_{3}}{k_{-1} k_{-2}+k_{-1} k_{3}+k_{2} k_{3}}
$$

SH loss was also treated by applying a steady-state approximation to following reaction mechanism,

$$
\mathbf{1} \underset{k_{-4}}{\stackrel{k_{4}}{\rightleftharpoons}} \mathbf{4} \stackrel{k_{5}}{\rightarrow} \mathrm{D} 2
$$

which yields the following expression

$$
k_{\mathrm{SH} \mathrm{loss}}=\frac{k_{4} k_{5}}{k_{-4}+k_{5}}
$$

To calculate the rate constants for $k_{5}$ and $S$ loss, variational transition state theory (VTST) was used as these reaction pathways lack saddle points. For these calculations, the geometry was reoptimized with corresponding harmonic vibrational frequencies at defined $\mathrm{C}_{1}-\mathrm{S}$ bond lengths up to 4 $\AA$. For both of these $\mathrm{C}-\mathrm{S}$ bond cleavage reactions, the minimum rate was found near $3.5 \AA$, which is approximately $2 r_{\text {eq }}$. Using the equations above, the rate constants for each dissociation channel were calculated as a function of photon energy from 4.5 to $8 \mathrm{eV}$ and are plotted in Figure 6.

The rate constants for each channel at the experimental dissociation energies are listed in Table 3 along with the RRKM and experimental branching ratios. Values for the individual rate constants can be found in the Supporting Information (SI-3).

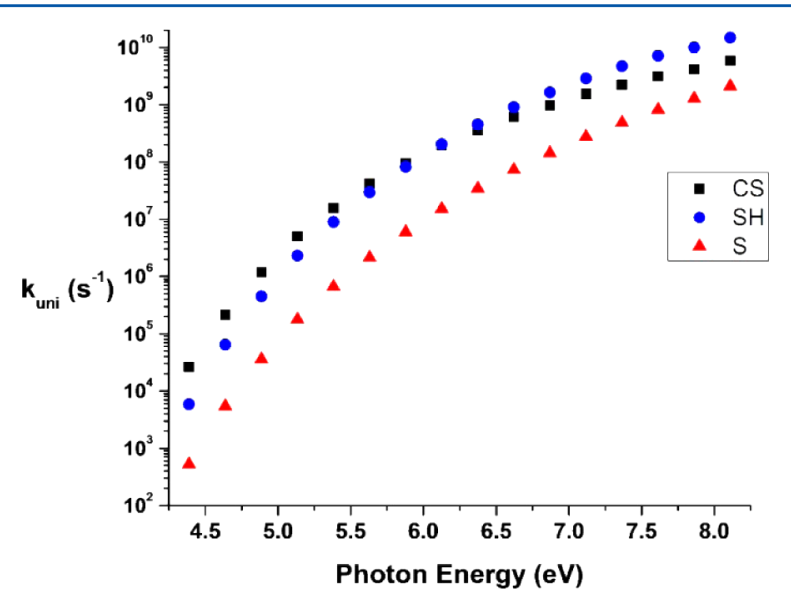

Figure 6. Unimolecular rate constants $\left(\mathrm{s}^{-1}\right)$ for each dissociation channel as a function of photon energy $(\mathrm{eV})$. 
Table 3. Calculated RRKM Rate Constants $\left(\mathrm{s}^{-1}\right)$ for Both Dissociation Channels and Photon Energies $(\mathrm{eV})$ with Corresponding Theoretical and Experimental Product Branching Ratios

\begin{tabular}{cccccc}
$\begin{array}{c}\text { photon } \\
\text { energy }\end{array}$ & $k_{\mathrm{CS}}$ & $k_{\mathrm{SH}}$ & $k_{\mathrm{S}}$ & $\begin{array}{c}\text { SH+S:CS } \\
(\mathrm{RRKM})\end{array}$ & $\begin{array}{c}\text { mass } \\
\text { channel } \\
\text { A:B (exp.) }\end{array}$ \\
5.0 & $3.2 \times 10^{6}$ & $1.2 \times 10^{6}$ & $7.7 \times 10^{4}$ & $0.40: 1$ & $0.30: 1$ \\
6.4 & $4.4 \times 10^{8}$ & $6.1 \times 10^{8}$ & $4.0 \times 10^{7}$ & $1.48: 1$ & $0.92: 1$ \\
7.9 & $4.6 \times 10^{9}$ & $1.1 \times 10^{10}$ & $1.4 \times 10^{9}$ & $2.69: 1$ & $1.44: 1$ \\
\hline
\end{tabular}

\section{DISCUSSION}

The primary goal of this study is to determine whether the photodissociation of thiophenoxy results in dissociation on an excited state or by decay via nonadiabatic interactions to the ground state followed by statistical dissociation. This question can be addressed by comparing experimentally derived $P\left(E_{\mathrm{T}}\right)$ distributions and product branching ratios to those expected for statistical decay.

At both dissociation energies, the $P\left(E_{\mathrm{T}}\right)$ distributions peak at low translational energy that is significantly less than the maximum allowed $E_{\mathrm{T}}$ for a given channel. This is characteristic of ground state dissociation as opposed to dissociation on a repulsive excited state, which often results in much higher fractional translational energy release. ${ }^{48}$ To test more quantitatively for statistical dissociation, the barrierless $\mathrm{SH}$ and $\mathrm{S}$ loss channels (mass channel A) can be modeled with a prior distribution ${ }^{49,50}$ using the following equation

$$
P\left(E_{\mathrm{T}} \mid E_{\mathrm{av}}\right) \propto E_{\mathrm{T}}^{1 / 2} \cdot \rho_{\mathrm{vr}}\left(E_{\mathrm{av}}-E_{\mathrm{T}}\right)
$$

where $E_{\mathrm{av}}$ is the available energy above the dissociation threshold, and $\rho_{\mathrm{vr}}\left(E_{\mathrm{av}}-E_{\mathrm{T}}\right)$ is rovibrational density of states of the fragments. For the simple model used here, the rotational density of states was treated as a constant. For the vibrational degrees of freedom, a semiclassical approximation of the vibrational density of states $^{46}$ was used. Although the translational energy distributions for mass channel A have contributions from $\mathrm{SH}$ and $\mathrm{S}$ loss, the prior distributions illustrate that the two channels will have similar translational energy release within a statistical interpretation. The calculated distributions shown in Figure 7 are in reasonable agreement with the experimental translational energy distributions.

For CS loss, the $P\left(E_{\mathrm{T}}\right)$ distributions are consistent with the presence of an exit barrier to the formation of the $\mathrm{C}_{5} \mathrm{H}_{5}$ and $\mathrm{CS}$ products, resulting in more of the available energy going into translation. We find that the highest energy transition state is the final transition state TS3 where the CS moiety departs from the cyclopentadienyl ring. TS3 presents a calculated barrier of $0.21 \mathrm{eV}$ with respect to products. The $P\left(E_{\mathrm{T}}\right)$ distributions for CS loss would then suggest that much of this energy goes into translation as is expected for the late "product-like" transition state found in the calculations.

The product branching ratios provide additional insight into the dissociation mechanism. They can be readily calculated within a statistical model using the RRKM calculations discussed in Section 3C. Within this framework, the branching ratios will be a function of both vibrational frequencies of the transition state and the available energy above the reaction barrier for each channel. For both the $\mathrm{SH}$ and $\mathrm{S}$ loss reaction pathways, C-S bond cleavage proceeds through a "loose" transition state where the $\mathrm{C}-\mathrm{S}$ bond lengthens. This is in contrast to the CS loss pathway where passage through an
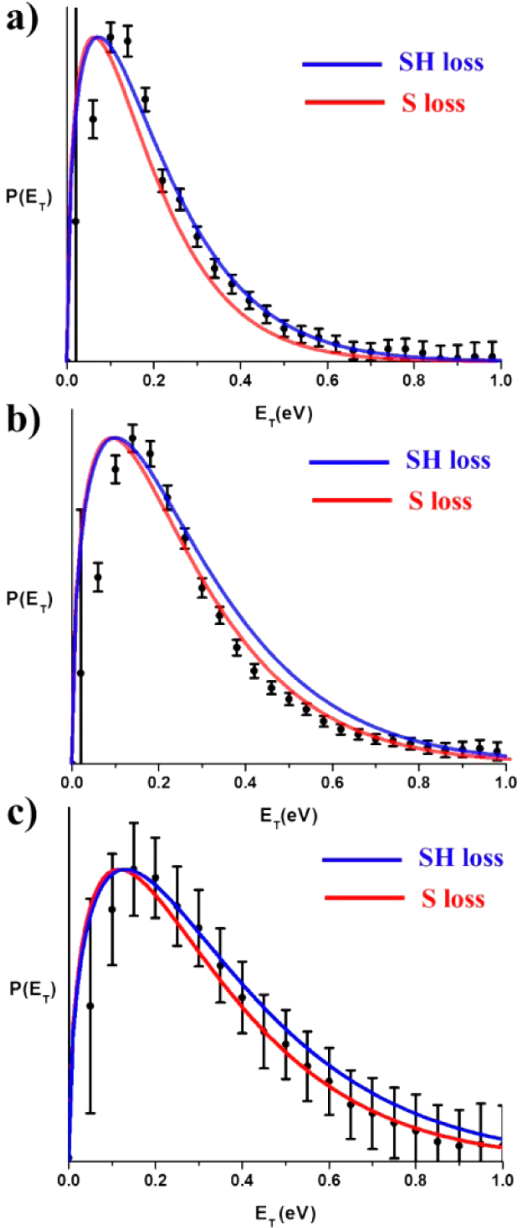

Figure 7. Calculated prior distributions for $\mathrm{SH}$ loss (blue) and $\mathrm{S}$ loss (red) at (a) 248, (b) 193, and (c) $157 \mathrm{~nm}$.

initial "tight" transition state is necessary for the formation of products. Although the reaction barrier for $\mathrm{SH}$ and $\mathrm{S}$ loss channels is higher than that for CS loss, the loose transition state for $\mathrm{SH}$ and $\mathrm{S}$ loss leads to a $k(E)$ that increases more rapidly as a function of the available energy. ${ }^{49}$ Indeed, we find experimentally that the relative population of the CS channel decreases significantly with photon energy from 248 to $157 \mathrm{~nm}$, where $\mathrm{SH} / \mathrm{S}$ loss (mass channel A) becomes more favorable than CS loss (mass channel B).

This trend is well-reproduced in the RRKM calculations. The calculations indicate that the branching ratios between mass channels A and B are largely determined by the relative rates of the SH and CS loss (Table 3 ) and that S loss should be minor at 248 and $193 \mathrm{~nm}$. However at $157 \mathrm{~nm}, \mathrm{~S}$ loss should be more competitive. This result is consistent with our simulations of mass distributions, which suggest that mass channel A is mostly SH loss at 248 and $193 \mathrm{~nm}$ but approximately 30\% S loss at 157 $\mathrm{nm}$.

At each of the wavelengths studied, the RRKM rates underestimate the contribution from CS loss in the measured branching ratios. Discrepancies in the calculated rates are likely due to error in the asymptotic energetics and in part to our assumption of harmonic vibrational modes for each dissociation channel. This assumption can be particularly problematic for the loose transition states associated with $\mathrm{SH}$ and $\mathrm{S}$ loss, since some vibrational modes might be better treated as hindered rotors. ${ }^{51}$ Nonetheless, the overall comparison of the exper- 
imental $P\left(E_{\mathrm{T}}\right)$ distributions and product branching ratios with expectations from statistical dissociation strongly suggest that the photodissociation of thiophenoxy proceeds via ground state statistical decay.

This conclusion then raises the question of how the photoexcited radical eventually finds its way to the ground electronic state. The excited states involved are still somewhat unclear. It is likely that at $248 \mathrm{~nm}(5.0 \mathrm{eV})$, there is population of the $3^{2} \mathrm{~B}_{1}$ state with a vertical excitation energy calculated to be approximately $4.2 \mathrm{eV}^{44}$ This state has strong oscillator strength and is believed to be responsible for the observed absorption of thiophenoxy near $300 \mathrm{~nm}$. Given that this is a highly excited electronic state of thiophenoxy with a manifold of lower-lying excited states, there are likely to be facile nonadiabatic pathways to the ground state via one or more conical intersections, leading to rapid internal conversion to the ground state. Population of the ground state prior to dissociation is further supported by the angular distributions which were found to be isotropic at each photon energy. Unfortunately, there are no electronic excited states above $5 \mathrm{eV}$ known for thiophenoxy that can be assigned to excitation at $193 \mathrm{~nm}(6.4 \mathrm{eV})$ and $157 \mathrm{~nm}(7.9 \mathrm{eV})$.

\section{CONCLUSIONS}

We have studied the photodissociation dynamics of thiophenoxy at 248, 193, and $157 \mathrm{~nm}$ by the means of fast beam coincidence translational spectroscopy. Our experiment yields the primary photochemistry for thiophenoxy photodissociation including the product branching ratios and translational energy distributions. SH loss and CS loss channels were identified as the major dissociation channels at each dissociation energy with $S$ loss becoming more competitive at $157 \mathrm{~nm}$. Electronic structure calculations show that on the ground electronic state, $\mathrm{SH}$ and S loss have no exit barrier with respect to final products. By contrast, the CS loss pathway, which occurs through multiple reactive intermediates and transition states, has a small exit barrier $(0.21 \mathrm{eV})$ between the final transition state and separated products. The measured translational energy distributions and product branching ratios are consistent with internal conversion to the ground electronic state followed by statistical dissociation.

\section{ASSOCIATED CONTENT}

\section{S Supporting Information}

Position resolution of the delay-line detector (SI-1), sample photofragment mass spectra simulation and estimated SH:S branching ratios (SI-2), values for the individual rate constants for CS and SH loss (SI-3), and multiphoton photodissociation data at $308 \mathrm{~nm}$ (SI-4). This material is available free of charge via the Internet at http://pubs.acs.org.

\section{AUTHOR INFORMATION}

\section{Corresponding Author}

*E-mail: dneumark@berkeley.edu.

\section{Present Addresses}

${ }^{\dagger}$ Korea Research Institute of Standards and Science, Daejeon 305-600, Republic of Korea.

${ }^{\ddagger}$ Department of Physics, Tsinghua University, Beijing, 100084, China.

\section{Notes}

The authors declare no competing financial interest.

\section{ACKNOWLEDGMENTS}

This research was supported by the Director, Office of Basic Energy Science, Chemical Sciences Division of the U.S. Department of Energy under Contract No. DE-AC0205CH11231. The authors would like to thank Thorsten Weber and Ottmar Jagutzki for their advice in setting up the delay-line detector and Hunter Shunatona for synthesizing deuterated thiophenol.

\section{REFERENCES}

(1) Graedel, T. E. The Homogeneous Chemistry of Atmospheric Sulfur. Rev. Geophys. 1977, 15, 421-428.

(2) Benson, S. W. Thermochemistry and Kinetics of SulfurContaining Molecules and Radicals. Chem. Rev. 1978, 78, 23-35.

(3) Cullis, C. F.; Mulcahy, M. F. R. The Kinetics of Combustion of Gaseous Sulphur Compounds. Combust. Flame 1972, 18, 225-292.

(4) Lim, J. S.; Lim, I. S.; Lee, K.-S.; Ahn, D.-S.; Lee, Y. S.; Kim, S. K. Intramolecular Orbital Alignment Observed in the Photodissociation of $\left[\mathrm{D}_{1}\right]$ Thiophenol. Angew. Chem., Int. Ed. 2006, 45, 6290-6293.

(5) Lim, I. S.; Lim, J. S.; Lee, Y. S.; Kim, S. K. Experimental and Theoretical Study of the Photodissociation Reaction of Thiophenol at $243 \mathrm{~nm}$ : Intramolecular Orbital Alignment of the Phenylthiyl Radical. J. Chem. Phys. 2007, 126, 034306-10.

(6) Lim, J. S.; Lee, Y. S.; Kim, S. K. Control of Intramolecular Orbital Alignment in the Photodissociation of Thiophenol: Conformational Manipulation by Chemical Substitution. Angew. Chem., Int. Ed. 2008, $47,1853-1856$

(7) Lim, J. S.; Kim, S. K. Experimental Probing of Conical Intersection Dynamics in the Photodissociation of Thioanisole. Nat. Chem. 2010, 2, 627-632.

(8) Devine, A. L.; Nix, M. G. D.; Dixon, R. N.; Ashfold, M. N. R. Near-Ultraviolet Photodissociation of Thiophenol. J. Phys. Chem. A 2008, 112, 9563-9574.

(9) Ashfold, M. N. R.; Devine, A. L.; Dixon, R. N.; King, G. A.; Nix, M. G. D.; Oliver, T. A. A. Exploring Nuclear Motion through Conical Intersections in the UV Photodissociation of Phenols and Thiophenol. Proc. Natl. Acad. Sci. U.S.A. 2008, 105, 12701-12706.

(10) Oliver, T. A. A.; King, G. A.; Tew, D. P.; Dixon, R. N.; Ashfold, M. N. R. Controlling Electronic Product Branching at Conical Intersections in the UV Photolysis of Para-Substituted Thiophenols. J. Phys. Chem. A 2012, 116, 12444-12459.

(11) Ito, O.; Matsuda, M. Evaluation of Addition Rates of pChlorobenzenethiyl Radical to Vinyl Monomers by Means of Flash Photolysis. J. Am. Chem. Soc. 1979, 101, 1815-1819.

(12) Nakamura, M.; Ito, O.; Matsuda, M. Substituent Effect on the Rate Constants for the Reactions between Benzenethiyl Radicals and Stable Free Radicals Estimated by Flash Photolysis. J. Am. Chem. Soc. 1980, 102, 698-701.

(13) Bonifacic, M.; Weiss, J.; Chaudhri, S. A.; Asmus, K. D. Oxidation of Thiols by Radical Cations of Organic Sulfides. J. Phys. Chem. 1985, 89, 3910-3914.

(14) Scott, T. W.; Liu, S. N. Picosecond Geminate Recombination of Phenylthiyl Free-Radical Pairs. J. Phys. Chem. 1989, 93, 1393-1396.

(15) Riyad, Y. M.; Naumov, S.; Hermann, R.; Brede, O. Deactivation of the First Excited Singlet State of Thiophenols. Phys. Chem. Chem. Phys. 2006, 8, 1697-1706.

(16) Zhang, Y.; Oliver, T. A. A.; Ashfold, M. N. R.; Bradforth, S. E. Contrasting the Excited State Reaction Pathways of Phenol and paraMethylthiophenol in the Gas and Liquid Phases. Faraday Disc. 2012, 157, 141-163.

(17) Thyrion, F. C. Flash Photolysis of Aromatic Sulfur Molecules. J. Phys. Chem. 1973, 77, 1478-1482.

(18) Porter, G.; Wright, F. J. Primary Photochemical Processes in Aromatic Molecules. Part 3. Absorption Spectra of Benzyl, Anilino, Phenoxy and Related Free Radicals. Trans. Faraday Soc. 1955, 51, $1469-1474$. 
(19) Tripathi, G. N. R.; Sun, Q.; Armstrong, D. A.; Chipman, D. M.; Schuler, R. H. Resonance Raman Spectra and Structure of Phenylthiyl Radical. J. Phys. Chem. 1992, 96, 5344-5350.

(20) Shibuya, K.; Nemoto, M.; Yanagibori, A.; Fukushima, M.; Obi, K. Spectroscopy and Kinetics of Thiophenoxy Radicals in the Gas Phase. Chem. Phys. 1988, 121, 237-244.

(21) Kim, J. B.; Yacovitch, T. I.; Hock, C.; Neumark, D. M. Slow Photoelectron Velocity-Map Imaging Spectroscopy of the Phenoxide and Thiophenoxide Anions. Phys. Chem. Chem. Phys. 2011, 13, $17378-17383$.

(22) Schmoltner, A. M.; Anex, D. S.; Lee, Y. T. Infrared Multiphoton Dissociation of Anisole: Production and Dissociation of Phenoxy Radical. J. Phys. Chem. 1992, 96, 1236-1240.

(23) Olivella, S.; Sole, A.; Garcia-Raso, A. Ab Initio Calculations of the Potential Surface for the Thermal Decomposition of the Phenoxyl Radical. J. Phys. Chem. 1995, 99, 10549-10556.

(24) Liu, R.; Morokuma, K.; Mebel, A. M.; Lin, M. C. Ab Initio Study of the Mechanism for the Thermal Decomposition of the Phenoxy Radical. J. Phys. Chem. 1996, 100, 9314-9322.

(25) Carstensen, H.-H.; Dean, A. M. A Quantitative Kinetic Analysis of CO Elimination from Phenoxy Radicals. Int. J. Chem. Kinet. 2012, 44, 75-89.

(26) Lin, C.-Y.; Lin, M. C. Thermal Decomposition of Methyl Phenyl Ether in Shock Waves: The Kinetics of Phenoxy Radical Reactions. J. Phys. Chem. 1986, 90, 425-431.

(27) Lias, S. G.; Bartmess, J. E.; Liebman, J. F.; Holmes, J. L.; Levin, R. D.; Millard, W. G. Gas Phase Ion and Neutral Thermochemistry; Journal of Physical and Chemical Reference Data Series; American Institute of Physics: New York, 1988; Vol. 17.

(28) Chase, M. W. NIST-JANAF Thermochemical Tables; NIST: Gaithersburg, MD, 1998, pp 1-1951.

(29) Nunes, P. M.; Agapito, F.; Costa Cabral, B. J.; Borges dos Santos, R. M.; Martinho Simoes, J. A. Enthalpy of Formation of the Cyclopentadienyl Radical: Photoacoustic Calorimetry and $\mathrm{Ab}$ Initio Studies. J. Phys. Chem. A 2006, 110, 5130-5134.

(30) Continetti, R. E.; Cyr, D. R.; Metz, R. B.; Neumark, D. M. Fast Beam Studies of $\mathrm{N}_{3}$ photodissociation. Chem. Phys. Lett. 1991, 182, 406-411.

(31) Hoops, A. A.; Gascooke, J. R.; Faulhaber, A. E.; Kautzman, K. E.; Neumark, D. M. Fast Beam Studies of $\mathrm{I}_{2}^{-}$and $\mathrm{I}_{2}^{-}$Ar Photodissociation. Chem. Phys. Lett. 2003, 374, 235-242.

(32) Bakker, J. M. B. A Beam-Modulated Time-of-Flight Mass Spectrometer I. Theoretical Considerations. J. Phys. E 1973, 6, 785789.

(33) Bakker, J. M. B. A Beam-Modulated Time-of-Flight Mass Spectrometer. II. Experimental Work. J. Phys. E 1974, 7, 364.

(34) Debruijn, D. P.; Los, J. Time and Position-Sensitive Detector for Dissociative Processes in Fast Beams. Rev. Sci. Instrum. 1982, 53, $1020-1026$

(35) Continetti, R. E.; Cyr, D. R.; Osborn, D. L.; Leahy, D. J.; Neumark, D. M. Photodissociation Dynamics of the $\mathrm{N}_{3}$ Radical. J. Chem. Phys. 1993, 99, 2616-2631.

(36) Zare, R. N. Photoejection Dynamics. Mol. Photochem. 1972, 4, $1-37$.

(37) Jagutzki, O.; Cerezo, A.; Czasch, A.; Dorner, R.; Hattas, M.; Min, H.; Mergel, V.; Spillmann, U.; Ullmann-Pfleger, K.; Weber, T.; et al. Multiple Hit Readout of a Microchannel Plate Detector with a Three-layer Delay-Line Anode. IEEE Trans. Nucl. Sci. 2002, 49, 24772483.

(38) Crider, P. E.; Harrison, A. W.; Neumark, D. M. Two- and Three-Body Photodissociation Dynamics of Diiodobromide $\mathrm{I}_{2} \mathrm{Br}^{-}$ Anion. J. Chem. Phys. 2011, 134.

(39) Harrison, A. W.; Lim, J. S.; Crider, P. E.; Neumark, D. M. Three-Body Photodissociation Dynamics of $\mathrm{I}_{2}^{-}\left(\mathrm{CO}_{2}\right)$. Chem. Phys. Lett. 2011, 512, 30-34.

(40) Lampton, M.; Siegmund, O.; Raffanti, R. Delay Line Anodes for Microchannel-Plate Spectrometers. Rev. Sci. Instrum. 1987, 58, 22982305 .
(41) Hanold, K. A.; Luong, A. K.; Clements, T. G.; Continetti, R. E. Photoelectron Multiple-Photofragment Coincidence Spectrometer. Rev. Sci. Instrum. 1999, 70, 2268-2276.

(42) Leahy, D. J.; Osborn, D. L.; Cyr, D. R.; Neumark, D. M. Predissociation Dynamics of the $\mathrm{O}_{2} \mathrm{~B}^{3} \Sigma_{\mathrm{u}}{ }^{-}$State: Vibrational State Dependence of the Product Fine-Structure Distribution. J. Chem. Phys. 1995, 103, 2495-2508.

(43) Cyr, D. R.; Leahy, D. J.; Osborn, D. L.; Continetti, R. E.; Neumark, D. M. Fast Beam Photodissociation of the $\mathrm{CH}_{2} \mathrm{NO}_{2}$ Radical. J. Chem. Phys. 1993, 99, 8751-8764.

(44) Cheng, C.-W.; Lee, Y.-P.; Witek, H. A. Theoretical Investigation of Molecular Properties of the First Excited State of the Thiophenoxyl Radical. J. Phys. Chem. A 2008, 112, 11998-12006.

(45) Frisch, M. J.; Trucks, G. W.; Schlegel, H. B.; Scuseria, G. E.; Robb, M. A.; Cheeseman, J. R.; Scalmani, G.; Barone, V.; Mennucci, B.; Petersson, G. A., et al. Gaussian 09, revision B.01; Gaussian, Inc.: Wallingford, CT, 2009.

(46) Marcus, R. A.; Rice, O. K. The Kinetics of the Recombination of Methyl Radicals and Iodine Atoms. J. Phys. Chem. 1951, 55, 894-908.

(47) Beyer, T.; Swinehart, D. F. Algorithm 448: Number of MultiplyRestricted Partitions. Commun. ACM 1973, 16, 379.

(48) Osborn, D. L.; Leahy, D. J.; Ross, E. M.; Neumark, D. M. Study of the Predissociation of $\mathrm{CH}_{3} \mathrm{O} \tilde{\mathrm{A}}\left({ }^{2} \mathrm{~A}_{1}\right)$ by Fast Beam Photofragment Translational Spectroscopy. Chem. Phys. Lett. 1995, 235, 484-489.

(49) Baer, T.; Hase, W. L. Unimolecular Reaction Dynamics: Theory and Experiments; Oxford University Press: New York, 1996.

(50) Leyh, B.; Gridelet, E.; Locht, R.; Lorquet, J. C. Analysis of Kinetic Energy Release Distributions by the Maximum Entropy Method. Int. J. Mass. Spectrom. 2006, 249-250, 330-339.

(51) Gilbert, R. G.; Smith, S. C. Theory of Unimolecular and Recombination Reactions; Blackwell Scientific Publications Journal: Oxford, U.K., 1990.

\section{NOTE ADDED AFTER ASAP PUBLICATION}

This manuscript was published ASAP on July 29, 2013. A correction was made to Table 3 and the revised version was reposted to the Web on August 1, 2013. 